\title{
Computer models of hydraulic systems of district heating
}

\author{
Anton Eremin ${ }^{1, *}$, Konstantin Trubitsyn, Sergey Kolesnikov, Igor Kudinov and Vasily \\ Tkachev
}

${ }^{1}$ Samara State Technical University, Molodogvardeyskaya 244, 443100, Samara, Russia

\begin{abstract}
A mathematical and computer model of a district heating network fed by two heat sources located at significantly different elevation marks has been developed. The model is based on the electrohydraulic analogy of electric current spread in conductors and liquid pressure spread in pipelines, which are described by the same equations. In particular, the first and second Kirchhoff's laws used in the calculation of electrical networks are applied to calculate the velocities and pressures in a complex multi-ring pipeline system. In order to maximize the approximation of the computer model to the real hydraulic network (in resistance to the process of heating agent flow), the method of automatic identification of the model is applied. This method is an iterative process of changing the hydraulic resistances in pipelines of the model in such a way that the results obtained from the calculations would have the least differences from the experimental data. The accuracy of identification depending on the number of points with known experimental data is $3-5 \%$.
\end{abstract}

\section{Introduction}

Known mathematical models for transfer processes of mass and pulses are extremely complex. The research is difficult when using not only accurate methods but also approximate analytical ones. Therefore, using the analogy method is particularly effective, as it allows performing a research of any physical process on objects of a completely different nature but described by identical differential equations. This area of research includes the theory of hydraulic networks which is described in papers [1 - 15]. And, in particular, paper [5] includes the sequence of Kirchhoff's laws in mathematical modelling for hydraulic systems. However, the application of this method for calculations of complex multi-ring branched pipeline systems is associated with a large amount of computation. To address these difficulties, iterative calculation methods based on the so-called «balancing flow rates» are used in this research $[1,3,4,6,8]$.

\section{Fundamental provisions of the proposed research method}

\footnotetext{
${ }^{*}$ Corresponding author: a.v.eremin@list.ru
} 
Consider a specific example of mathematical model design and find flow rates distribution in the net consisting of 1 ring and 3 branches. Flow rates in ring sections $a, b$, $c, d$ are shown as $Q_{a}, Q_{b}, Q_{c}, Q_{d}$, and in branches - as $Q_{1}, Q_{2}, Q_{3}$. It is required to find the flow rates distribution in ring sections (with given flow rates) at ring inlet. Medium flow rates in branches $Q_{1}, Q_{2}, Q_{3}$ are known, and their sum is equal to the flow rate at ring inlet.

The first Kirchhoff's law applied to hydraulic networks establishes the equality of the inflow and outflow of the medium in each unit

$$
\sum_{i=1}^{n} Q_{i}=0
$$

where $n$-number of pipelines in the unit; $Q_{i}(i=\overline{1, n})$ - flow rates in pipelines.

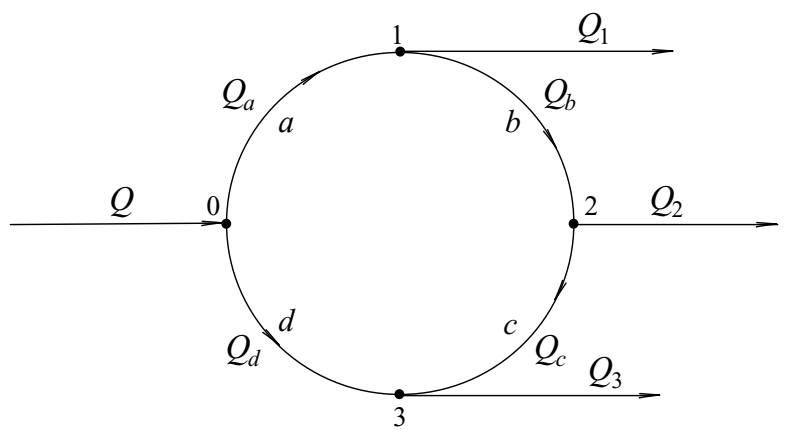

Fig. 1. Scheme of a single-ring pipeline network with three branches.

According to the second Kirchhoff's law, the sum of heads is equal to zero for any closedring system

$$
\sum_{i=1}^{n} H_{i}=\sum_{i=1}^{n} S_{i} Q_{i}^{2}=0,
$$

where $S_{i}(i=\overline{1, n})$ - hydraulic resistance of $i$-section.

Using relations (1), (2) by iterative calculation we can find the flow rates distribution in network sections at a certain flow rate $Q$, given at the ring inlet. At the first step of iteration, generalized distributions of flow rates $Q_{a}, Q_{b}, Q_{c}, Q_{d}$ are given in every section of a ring. Then for units $0,1,2$ according to the first Kirchhoff's law, we have $Q_{d}=Q-Q_{a}$; $Q_{a}=Q_{1}+Q_{b} ; Q_{b}=Q_{2}+Q_{c}$.

According to the second Kirchhoff's law on the basis of given flow rates in the sections of the ring there is a head discrepancy

$$
\delta H=\sum_{i=1}^{4} S_{i} Q_{i}^{2}=S_{a} Q_{a}^{2}+S_{b} Q_{b}^{2}+S_{c} Q_{c}^{2}-S_{d} Q_{d}^{2} .
$$

To approximate $\delta H$ to zero, the correction (balancing) flow rate $\delta Q$ is introduced which should be subtracted from the flow rate in overloaded sections and be added to under loaded ones. The value of the balancing flow rate can be found from relation (3), if $\delta H=0$. 


$$
S_{a}\left(Q_{a}-\delta Q\right)^{2}+S_{b}\left(Q_{b}-\delta Q\right)^{2}+S_{c}\left(Q_{c}-\delta Q\right)^{2}-S_{d}\left(Q_{d}+\delta Q\right)^{2}=0 .
$$

Neglecting terms containing $(\delta Q)^{2}$ as relatively small, the relation (4) with respect to the balancing flow rate $\delta Q$ is reduced to an algebraic linear equation. Its solution is

$$
\delta Q=\delta H /\left(2 \sum_{i=1}^{n} S_{i} Q_{i}\right)
$$

where $\sum_{i=1}^{n} S_{i} Q_{i}=S_{a} Q_{a}+S_{b} Q_{b}+S_{c} Q_{c}+S_{d} Q_{d}$.

Having calculated $\delta Q$, the flow rates in ring sections are to be refined. Iterative calculations continue until the flow rates of the last two iterations differ by a specified value.

As an example, let's find the solution of a specific problem of flow rates distribution for sections of the ring network with the initial data: $Q=60 \mathrm{~m} / \mathrm{h} ; S_{a}=5 \cdot 10^{-5} \mathrm{~h}^{2} / \mathrm{m}^{5}$; $S_{b}=2 \cdot 10^{-5} \mathrm{~h}^{2} / \mathrm{m}^{5} ; S_{c}=8 \cdot 10^{-5} \mathrm{~h}^{2} / \mathrm{m}^{5} ; S_{d}=4 \cdot 10^{-5} \mathrm{~h}^{2} / \mathrm{m}^{5} ; Q_{1}=15 \mathrm{~m}^{3} / \mathrm{h} ; Q_{2}=25 \mathrm{~m}^{3} / \mathrm{h} ;$ $Q_{3}=20 \mathrm{~m}^{3} / \mathrm{h}$.

At the first step of iteration, we call the following (arbitrary) flow rates in network sections: $Q_{a}=50 \mathrm{~m}^{3} / \mathrm{h} ; Q_{b}=35 \mathrm{~m}^{3} / \mathrm{h} ; Q_{c}=10 \mathrm{~m}^{3} / \mathrm{h} ; Q_{d}=10 \mathrm{~m}^{3} / \mathrm{h}$.

Using formula (3), we find $\delta H=0.1535 \mathrm{~m}$. The balancing flow rate determined by formula (5), will be $\delta Q=17.47 \mathrm{~m}$.

At the second iteration step, the following flow rates are given in the sections of the ring network $Q_{a}=32.53 \mathrm{~m}^{3} / \mathrm{h} ; Q_{b}=17.53 \mathrm{~m}^{3} / \mathrm{h} ; Q_{c}=-7.47 \mathrm{~m}^{3} / \mathrm{h} ; Q_{d}=27.47 \mathrm{~m}^{3} / \mathrm{h}$.

The flow rate sign $Q_{c}$ in this case is negative. Consequently, the direction of medium motion given at the first step of iteration in this section must be reversed. Then the flow rate can be considered as positive. Hence, we have $\delta H=0.024408 \mathrm{~m} ; \delta Q=3.32 \mathrm{~m}^{3} / \mathrm{h}$.

Making more exact the flow rates in the sections, we find $Q_{a}=29.21 \mathrm{~m}^{3} / \mathrm{h}, Q_{b}=14.21$ $\mathrm{m}^{3} / \mathrm{h}, Q_{c}=10.79 \mathrm{~m}^{3} / \mathrm{h}, Q_{d}=30.79 \mathrm{~m}^{3} / \mathrm{h}$.

By formulas (3) and (5) we find the values $\delta H$ and $\delta Q$ at the third step of the iteration $\delta H$ $=0.0074 \mathrm{~m} ; \delta Q=1.1 \mathrm{~m}^{3} / \mathrm{h}$.

Based on the results of the third iteration, we specify the flow rates in the sections of the ring network which will be $Q_{a}=28.11 \mathrm{~m}^{3} / \mathrm{h}, Q_{b}=13.11 \mathrm{~m}^{3} / \mathrm{h}, Q_{c}=11.89 \mathrm{~m}^{3} / \mathrm{h}, Q_{d}=31.89 \mathrm{~m}^{3} / \mathrm{h}$.

Performing the test of equation of the first Kirchhoff's law for all units of the network, we find $Q_{1}=15 \mathrm{~m}^{3} / \mathrm{h} ; \quad Q_{2}=25 \mathrm{~m} / \mathrm{h} ; \quad Q_{3}=20 \mathrm{~m}^{3} / \mathrm{h} ; \quad Q_{1}=Q_{a}-Q_{b}=15 \mathrm{~m}^{3} / \mathrm{h}$; $Q_{2}=Q_{b}+Q_{c}=25 \mathrm{~m}^{3} / \mathrm{h} ; Q_{3}=Q_{d}-Q_{c}=20 \mathrm{~m}^{3} / \mathrm{h}$. Thus, already at the third step of iteration, the flow rates for the sections of the ring network that satisfy the specified flow rates for $Q_{1}, Q_{2}, Q_{3}$ are found.

In case of multi-ring branched hydraulic networks, the implementation of the above calculation algorithm is possible only with the use of the computer technology.

So, it is necessary to design a computer model of the load-flow process. Kirchhoff's laws (1), (2), as well as the theory of graphs [7 - 11] develop the basis of its design resulting in a «tree» of the heat network (Fig. 2).

Let us call the vertices (units) of the graph by the numbers $1,2,3, \ldots, 9$, and the arcs - by letters $a, b, c, \ldots$ (Fig. 2). The vertices of the graph are the connection points of pipelines, 
and the arcs are their sections. The «tree» is constructed so that any vertex can be reached from vertex 1 .

Every vertex is marked with the number, height of the location, the value of inflow or outflow of the medium. Every arc has the number, the length and the diameter of pipes, friction factor, etc. In this way, the heat network is presented as a single hydraulic system, all areas of which are interconnected. Thus, it is possible to perform Pascal's law and the equation of continuity of the flow.

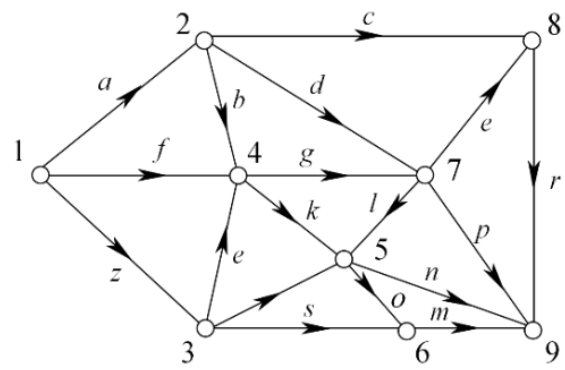

Fig. 2. Scheme of the heat network.

With the help of relations (3), (5) we can build a closed equation system for relatively unknown flow rates in the sections of the network and pressures at its units. By iterative method of calculation applied to complex multi-ring networks, the problem of iterations convergence occurs. In computational practice, the most common method is the full-system pressure drop balancing. According to this method, a certain initial approximation for flow rates in all sections is given; pressure losses and total discrepancies are found. These discrepancies determine the values of so-called «balancing flow rates»; a minimum of discrepancies within the specified values is found. Note that the method of full-system pressure drop balancing is characterized by rapid iteration convergence $[1-3,5,8]$.

Thus, the «tree» of the heat network includes units and sections. The units may be with a given flow rates (the heating agent is taken from the unit) and with a given inflow (the heating agent enters the unit). The sections are divided into: sections-pipes; sections-pumps; sectionsvalves and sections-heat exchangers. When designing a network model it is necessary to find hydraulic characteristics (functions of head losses from the flow rate) in all sections. For example, head losses in a pipeline include linear losses and local losses

$$
\Delta h=\lambda \frac{l}{d} \frac{v^{2}}{2 g}+\sum \xi \frac{v^{2}}{2 g},
$$

where $\Delta h$ - head loss, $\mathrm{m} ; \lambda$ - flow friction factor; $l$ - length of the pipeline, $\mathrm{m} ; d$-inner diameter, $\mathrm{m} ; v$ - average speed, $\mathrm{m} / \mathrm{s} ; \sum \xi$ - sum of local friction factors in the section; $g$ - intensity of gravity, $\mathrm{m} / \mathrm{s}^{2}$.

Head losses in local resistances through an equivalent length are reduced to linear equations with the calculation of the equivalent length by the formula $l_{\mathrm{e}}=d \sum \xi / \lambda$. From here, the formula (6) will be

$$
\Delta h=\frac{v^{2}}{2 g}\left(\lambda \frac{l}{d}+\lambda \frac{l_{e}}{d}\right)=\frac{\lambda v^{2}}{2 g}\left(l+l_{e}\right) .
$$


Considering that $\mathrm{v}=4 Q /\left(\pi d^{2}\right)$, we find $\Delta h=8 \lambda Q^{2}\left(l+l_{e}\right) /\left(\pi^{2} g d^{5}\right)$. Hydraulic characteristic of the section-pipe will be $\Delta h=s Q^{2}$, where $s=8 \lambda\left(l+l_{e}\right) / /\left(\pi^{2} g d^{5}\right)$ hydraulic resistance of the section, $\mathrm{s}^{2} / \mathrm{m}^{2}$.

For each section-pipe we introduce such parameters as diameter; length and factors of local resistances. For the valve-section, the hydraulic characteristic will be $\Delta h=s Q^{2}$, where $s$ - factor (for full-opening valve which is 0.07 ).

Sections-pumps in the model are presented by the dependencies between the head of the pump and its feed. The characteristic of the pump is determined by the formula $H=H_{\phi}-Q_{\mathrm{H}}^{2} S_{\phi}$, where $H_{\phi}$ - shut-off head at outlet $Q_{\mathrm{H}}=0 ; Q_{\mathrm{H}}-$ pump feed; $S_{\phi}-$ hydraulic pump resistance.

Above algorithm provides building a model with the passport specifications. However, the specifications of a real network may differ significantly from the passport ones. For maximum approximation of the computer model to the real network, the model identification is performed $[1-4,6,8,12]$. For this purpose, experimental pressure values are used in discrete points of the network. To approximate the model to a real system, the hydraulic resistances of the sections are altered so that the pressures obtained on the model differ as little as possible from the experimental data. The identification process is iterative, and it is automated in the model. Its accuracy is determined by the number of points with given experimental data.

In particular, specific calculations show that at known experimental pressures at 60 points of the heating network (for a model having 210 unit points), the accuracy of identification is $3-5 \%$.

After performing the identification, the computer model of the real heat network may be considered to be built. Such a model allows performing almost any number of computational experiments with respect to a given heat network. The advantage of this model is the ability to carry out any changes in the network in order to eliminate existing problems which allows changing the current mode, and also choosing the best options for the reconstruction of the heating network.

\section{Discussion of the results}

Analysis of the results obtained for a number of objects shows the problems that are common to many heating systems, so the ways of solving them will be practically identical. In this regard, the heating network (Fig. 3) is an indicative example.

Consider the data on the flow rates characteristics for heat removal pipes at CHP-1 (combined heat and power plant) and CHP-2. The total water flow rate from CHP-1 is 12000 $\mathrm{t} / \mathrm{h}$ in direct pipelines and $10000 \mathrm{t} / \mathrm{h}$ in return pipelines where the first heat removal pipe is $4200 \mathrm{t} / \mathrm{h}$ in the direct pipeline and $1550 \mathrm{t} / \mathrm{h}$ in the return one. The second heat removal pipe is $7100 \mathrm{t} / \mathrm{h}$ in the direct pipeline and $8200 \mathrm{t} / \mathrm{h}$ in the return one. The total water flow rate from CHP-2 is $7300 \mathrm{t} / \mathrm{h}$ in the direct pipelines and $6500 \mathrm{t} / \mathrm{h}$ in the return ones where the first heat removal pipe is 830 and $650 \mathrm{t} / \mathrm{h}$, the second -680 and $590 \mathrm{t} / \mathrm{h}$, the third -4060 and $3620 \mathrm{t} / \mathrm{h}$, the fourth -1430 and $1150 \mathrm{t} / \mathrm{h}$ respectively.

The results of piezometric pressure calculations for all heat removal pipes at CHP-1 and CHP-2 in the real mode of their operation are given in fig. $4-9$. Let's analyse the results obtained. An important feature of the heat networks under study is a significant difference in elevation marks of heat sources locations. In particular, the elevation mark of CHP-1 is 85 $\mathrm{m}$, and CHP-2 is $32 \mathrm{~m}$. Here, the heat networks powered from CHP-1 and CHP-2 are completely separated by closed valves (fig. 3). 
The most important thing is that CHP-1(elevation mark $85 \mathrm{~m}$ ) feeds consumers located near CHP-2 (elevation mark $33 \mathrm{~m}$ ) (Fig. 3, 4 the first heat removal pipe at CHP-1). Consumers are located at a distance of about $16 \mathrm{~km}$ from CHP-1. In addition, at a distance of about 3 to $5 \mathrm{~km}$ from CHP-1 there is a section of the heating network with an elevation mark of about $167 \mathrm{~m}$. Therefore, the piezometric pressure in the return pipeline should be maintained at a level not lower than $180 \mathrm{~m}$, which is provided by the corresponding supporting valves (V24, V26, fig. 3, 4, 5).

Also, CHP-2 (elevation mark $32 \mathrm{~m}$ ) supplies consumers located at $113 \mathrm{~m}$. To raise water at this altitude, it is necessary to use boosting pump stations (in the CHP-2 scheme there are three such pumping stations HC-4, HC-5 and HC-10). Consequently, it is more reasonable to supply these consumers from CHP-1 but consumers located at elevations of $30-50$ meters from CHP-2. In this connection, it is possible to propose the following scheme for the supply of consumers from CHP-1 and CHP-2 (in fig. 3 a division scheme is marked by a wavy line).

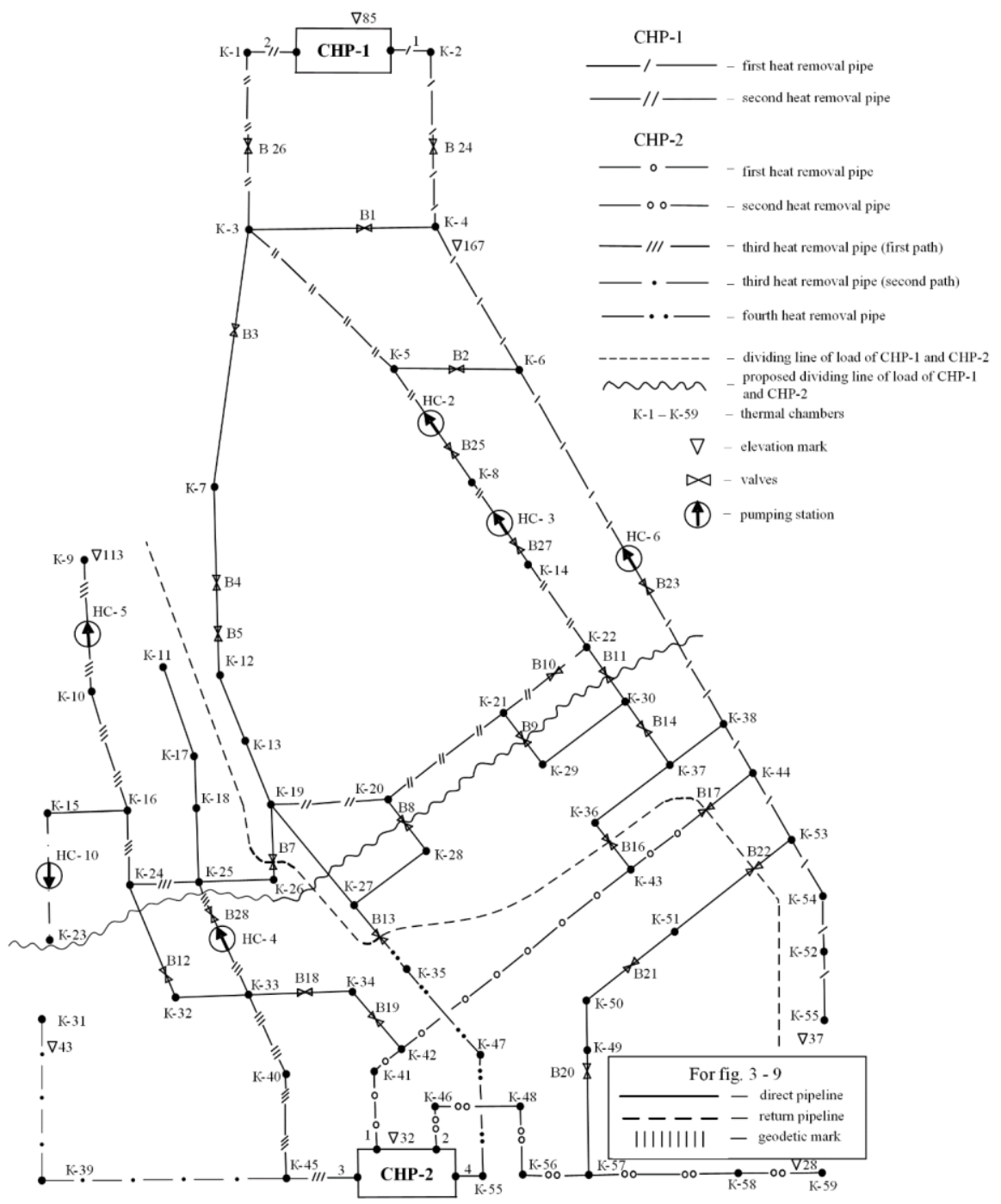

Fig. 3. Scheme of heat networks from CHP-1 and CHP-2. 
Analysis of the results of piezometric pressure calculations for the first heat removal pipe at CHP-1 (Fig. 4) allows us to conclude that in the section of the K-55 thermal chamber, the available pressure difference between the direct and return pipelines is about $10 \mathrm{~m}$ of $\mathrm{H} 2 \mathrm{O}$ (minimum permissible (passport) is $20 \mathrm{~m}$ of $\mathrm{H} 2 \mathrm{O}$ ). To solve this problem, it is necessary to reduce the pressure throttling in the direct pipeline by the appropriate value with the help of the valve V23. In a similar way, it is possible to solve the problem of insufficient available pressure drop in the section of the K-19 thermal chamber of the second heat removal pipe at CHP-1 (fig. 5), reducing the pressure throttling in the direct pipeline with the help of the valve V27. Thus, in order to ensure the required available differential pressure, it is only necessary to properly adjust the joint operation of the throttling valves in direct pipelines and in step-down pump stations in return pipelines which can effectively be performed on a computer model.

Diagrams of pressures in heat removal pipe at CHP-2 are shown in fig. $6-9$. Their analysis allows us to conclude that the main problem at almost all heat removal pipes is the insufficient available differential pressure. For example, in the first path of the third heat removal pipe (fig. 8), it is only about $8 \mathrm{~m} \mathrm{H} 2 \mathrm{O}$ (in the section of the thermal chamber K-31), and in the section of the K-16 thermal chamber of the second path of the third heat removal pipe (fig. 9) there is an intersection of the piezometric lines of the direct and return pipelines. The main reason is the insufficient head generated by the network pumps of the internal circuit of the heating network of CHP-2 which is $51 \mathrm{~m} \mathrm{H} 2 \mathrm{O}$. Calculations on the model showed that an increase in the pressure (by $20-30 \mathrm{~m} \mathrm{H} 2 \mathrm{O}$ ) generated by the network pumps of CHP-2 will make it possible to take in practically all the existing and planned load for the heating system from CHP-2 without any reconstruction of the heating network pipelines.

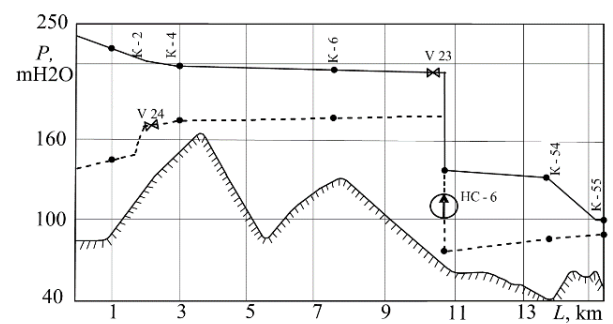

Fig. 4. The first heat removal pipe at CHP-1: $P$ - piezometric pressure. $L$ - length of pipelines.

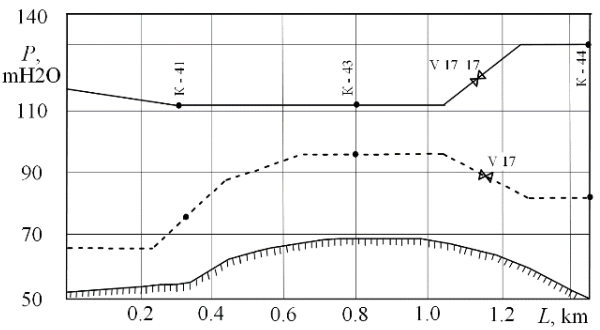

Fig. 6. The first heat removal pipe at CHP-2.

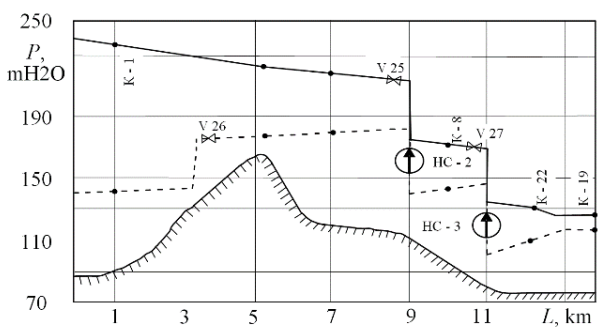

Fig. 5. The second heat removal pipe at CHP-1. HC-2, HC-3 - step-down pumping stations.

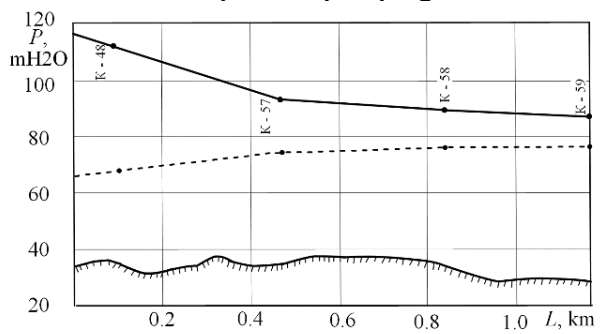

Fig. 7. The second heat removal pipe at CHP-2. 


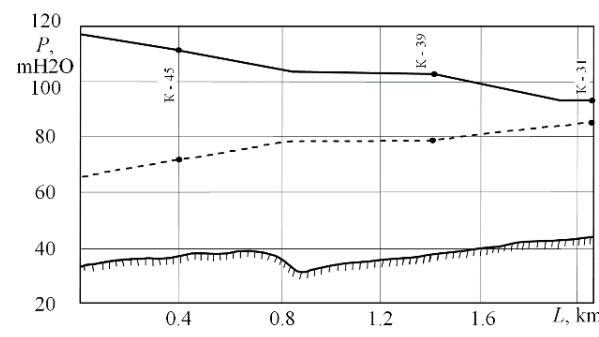

Fig. 8. The third heat removal pipe at CHP-2 (the first path).

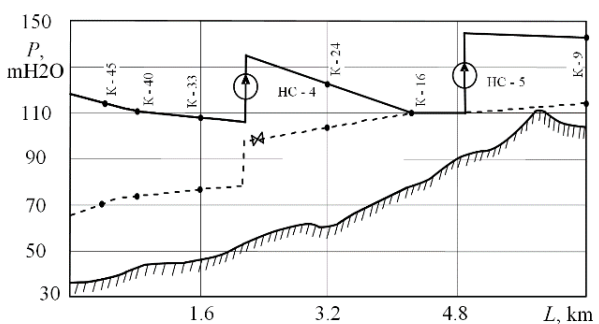

Fig. 9. The third heat removal pipe at CHP-2 (the second path). HC-4, HC-5 - boosting pumping.

It is necessary to transfer the entire load of consumers located at elevation marks of 30 to $50 \mathrm{~m}$ to CHP-2, and the load of consumers at elevation marks of more than $60 \mathrm{~m}$ to CHP-1. The studies performed on a computer model showed that such a redistribution of the load in the existing heat network scheme is possible under conditions of reconstruction of pipelines in the section from K-19 to K-26 with a diameter change from 400 to $800 \mathrm{~mm}$ (the length of the section is about $1.1 \mathrm{~km}$ ). Therein, there is no need to use the boosting pumping stations HC-4, HC-5, HC-10 and the step-down pumping station HC-6.

\section{Conclusions}

1. A mathematical and computer model of the heat network from two heat sources - CHP-1 and CHP-2 was designed. It allows determining the pressure, flow rate and flow velocity of the heating agent at any point of the network, as well as head losses and energy costs for moving the medium. In order to maximize the model's approximation to the real network the automatic identification is performed which is an iterative process of changing the pipeline network resistances using experimental data on pressures at discrete points.

2. In heating networks with large differences in elevation marks of the source and the consumer it is normally to use boosting and step-down pumping stations. In this case, problems can arise not because of large head losses in some sections of the network because of insufficient diameter of the pipelines, but as a result of improper adjustment of the joint operation of step-down (boosting) pumping stations and throttling valves in direct pipelines and retaining valves in return ones.

\section{Acknowledgments}

The research was performed with the financial support of the Ministry of Education and Science of the Russian Federation within the framework of the basic part of the Governmental Task of the Federal State Budgetary Educational Institution of Higher Education SSTU (Samara State Technical University) (project No.1.5551. 2017/8.9).

\section{References}

1. A.G. Kovalenko, V.A. Kudinov, The program for modelling of hydraulic and thermal modes of heating systems at CHP and heat supply, Registered in the registry of computer programs № 2007611682 (2007)

2. V.A. Kudinov, A.G. Kovalenko, S.V. Kolesnikov, Yu.S. Panamarev, Izvestiya Akademii Nauk. Energetika 6 (2001)

3. N.A. Zroychikov, V.A. Kudinov, A.G. Kovalenko, S.V. Kolesnikov, A.G. Moskvin, V.I. Lisitsa, Teploenergetika 54 (2007) DOI: 10.1134/S004060150711002X 
4. I.V. Kudinov, S.V. Kolesnikov, A.V. Eremin, A.N. Branfileva, Thermal Engineering 60(11) (2013) DOI: 10.1134/S0040601513080053

5. E.Y. Sokolov, Heating and heating networks (1982)

6. A.P. Merenkov, V.Y. Khasilev, The theory of hydraulic circuits (1985)

7. S.V. Kolesnikov, Vestnik of Samara State Technical University, series of Technical sciences 2(42) (2014)

8. A.P. Merenkov, Journal of Computational Mathematics and Mathematical Physics 13, 5 (1973)

9. A.P. Merenkov, Izvestita AN SSSR Power industry and transport 4 (1963)

10. A.P. Merenkov, Izvestita AN SSSR Power industry and transport 6 (1971)

11. M.G. Sukharev, Izvestiya Vuzov Oil and gas 6 (1965)

12. I.V. Kudinov, V.A. Kudinov, A.V. Eremin, Journal of Engineering Physics and Thermophysics 86(2) (2013) DOI: 10.1007/s10891-013-0849-4

13. S.V. Sumarokov, Mathematical modeling of water supply systems (1983)

14. S.V. Sumarokov, Economics and Mathematical Methods 12(5) (1973)

15. A.A. Zykov, The theory of finite graphs (1969) 